\title{
HENRI HUPNER
}

\section{$1891-1958$}

\section{A}

U lendemain des $V^{e s}$ Journées de l'Hydraulique, où plusieurs de nos Collègues l'avaient vu participer aux séances et présider deux d'entre elles, nous avons eu la douleur d'ap. prendre la mort rapide de $M$. Hupner

A ses obsèques où la S.H.F. était représentée, $M$. Bernard Renaud, vice-président du Conseil Général des Ponts et Chaussées, a retracé sa carrière, qui le conduisit jusqu'aux fonctions d'Inspecteur Général, chargé du Service Technique des Grands Barrages et M. Louis Saulgeot, directeur de l'Electricité et du Gaz au Ministère de l'Industrie et du Commerce, a évoqué les divers postes administratifs où il s'était occupé plus particulière. ment, de l'énergie et de la construction électriques.

Nous empruntons à ces nécrologies une grande partie des lignes qui suivent.

Henri Hupner était né le 18 mai 1891 à Paris, d'une famille ardennaise. Après de fortes études au collège Chaptal, il fut reçu dans un très bon rang à l'Ecole Polytechnique au concours de 1911.
Malgré son inaptitude reconnue au service militaire, il fit preuve en toute occasion de ce patriotisme gravé au cœur des enfants des Marches de 1'Est : en 1914, affecté, comme élève-ingénieur des Ponts et Chaussées au Service de la Navigation, à Reims, il n'hésite pas à faire maintes tournées sur la ligne de combat et, en 1940, appelé à la direction du Service ordinaire du département des Ardennes, “ il se dépensa sans compter pour apporter son concours aux armées françaises en opération dans la vallée de la Meuse, fournit au commissaire militaire l'appui le plus dévoué pour assurer le repli du matériel flottant; ... ne se retirant qu'à proximité immédiate de l'ennemi ) (nous citons le texte même de sa citation à l'ordre du

Studio Harcourt
Corps d'Armée).

Sa carrière administrative devait témoigner de ses grandes qualités : zèle méticuleux, avidité de connaître, ingéniosité inlassable à faire le bien, grande modestie.

Dans les postes qu'il occupa, à Rouen comme ingénieur de 1920 à 1930 et à Agen comme ingé- 
nieur en chef, de 1940 à 1943, M. Hupner sut mener à bien des travaux de construction de plusieurs ponts : sur la Seine, sur la Garonne et sur le Lot. Il rédigea, à cette occasion, pour les Annales des Ponts et Chaussées un article très remarqué sur l'application des séries trigonométriques à l'étude du flambement des arcs. Il se distingua, également par le zèle qu'il déploya à Agen au cours des graves inondations de 1935.

Promu Inspecteur Général des Ponts et Chaussées le $1^{\text {er }}$ septembre 1943, il fut détaché dans les fonctions particulièrement délicates de Répartiteur Chef de la Section d'Electricité de l'Office Central de Répartition des Produits industriels, puis dans celles de Commissaire du Comité d'Organisation des Produits de Carrières et de Dragages et des Chaux et Ciments. Il remplit ces fonctions avec le doigté et la réussite qu'il obtient dans tout ce dont il est chargé, comme, aussi, dans les superarbitrages de conflits sociaux, qui lui sont maintés fois confiés par l'Administration.

Nommé chef du Service des Grands Barrages en 1947, il participa à l'élaboration des projets de tous les grands ouvrages hydrauliques, tels que Bort, Tignes, Donzère-Mondragon, et bien d'autres, dont la renommée et le prestige s'étendent au-delà de nos frontières. Il y applique toute l'acuité d'observation, la rigueur nécessaire aux garanties de la sécurité publique et, en même temps, la compréhension qui le caractérisent. $M$ ais son activité l'entraîne bien au-delà de ces fonctions, déjà très lourdes.

A partir de 1948, il prend une part des plus importantes à l'équipement hydroélectrique de l'Algérie.

Sa participation au Congrès des Grands Barrages à Stockholm en 1948, au Congrès des Recherches Hydrauliques à Bombay en 1951, à la Commission d'Hydraulique fluviale des Nations-Unies en 1951, sa nomination comme chef de la Délégation française au Congrès International des Grands Barrages à New Delhi en 1951 contribuent, de la manière la plus marquante, au relief de la représentation française dans ces réunions.

Il avait, ancrée au fond de lui-même, la passion de connaître, et peu de domaines échappaient à son investigation. Il fut, en effet, président de la Société française de Météorologie en 1950-1951, membre de la Réunion internationale des Laboratoires d'Essais de Matériaux, délégué de la
France à la Mission internationale aux EtatsUnis, organisée par l'O.E.C.E. à l'occasion du Cinquantenaire de la Société des Ingénieurs Civils américains, associé aux travaux du Conseil Supérieur de la Recherche Scientifique, membre du Comité Technique de 1'Energie des Mers, et membre du Comité Consultatif des Travaux d'Infrastructure pétrolière du Sahara.

$$
\text { is }
$$

Sa participation à l'activité de la Société Hydrotechnique de France était très étendue : nommé membre du Comité Technique en 1947, du Conseil d'administration en 1948, il était, depuis son entrée à notre société, président de la section "Génie civil et Conduites", et, depuis 1948 , président de la nouvelle Commission pour l'Etude des Débits de Crues, enfin, membre du Bureau du Comité Technique depuis la création, en 1955, de ce Bureau par $M$. Gibrat.

Assidu à toutes nos séances, répondant avec dévouement et affabilité à toutes les sollicitations, soit pour présider une séance, soit pour organiser une réunion, soit pour rendre compte d'un congrès ou d'une mission, $M$. Hupner participait, en outre, très fréquemment, aux discussions avec une grande culture et un sens très averti.

Enfin, il exerça la présidence de notre Société de juin 1954 à juin 1955.

En évoquant, à la réunion de notre Conseil d'administration du 17 octobre 1958, son inlassable activité et sa haute compétence longuement et pleinement consacrées au bien public, M. Gariel, alors président de la Société Hydrotechnique de France, les a résumées admirablement d'un mot, en qualifiant $M$. Hupner de modèle des "Grands Serviteurs de l'Etat ".

Pour sa part, M. Gibrat, président de notre Comité Technique, après avoir rendu à $M$. Hupner un touchant hommage en ouvrant la session des 20 et 21 novembre, s'est plu à faire siennes les paroles de $M$. Saulgeot à ses obsèques :

"Sur toutes ces qualités intellectuelles règne la lumière d'une rayonnante bonté qui lui attire la profonde affection de tous ceux qui l'approchent. Il est l'homme des petites attentions délicates. )

La disparition de $M$. Hupner laisse un grand vide dans l'esprit et dans le cour de notre Société. 